\title{
Identification of Open-Switch and Short-Switch Failure of Multilevel Inverters through DWT and ANN Approach using LabVIEW
}

\author{
E. Parimalasundar ${ }^{\dagger}$ and N. Suthanthira Vanitha*
}

\begin{abstract}
In recent times, multilevel inverters are given high priority in many large industrial drive applications. However, the reliability of multilevel inverters are mainly affected by the failure of power electronic switches. In this paper, open-switch and short-switch failure of multilevel inverters and its identification using a high performance diagnostic system is discussed. Experimental and simulation studies were carried out on five level cascaded H-Bridge multilevel inverter and its output voltage waveforms were analyzed at different switch fault cases and at different modulation index values. Salient frequency domain features of the output voltage signal were extracted using the discrete wavelet transform multi resolution signal decomposition technique. Real time application of the proposed fault diagnostic system was implemented through the LabVIEW software. Artificial neural network was trained offline using the Matlab software and the resultant network parameters were transferred to LabVIEW real time system. In the proposed system, it is possible to precisely identify the individual faulty switch (may be due to open-switch (or) short-switch failure) of multilevel inverters.
\end{abstract}

Keywords: Multilevel inverter, LabVIEW, DWT, Artificial neural networks, Fault diagnosis

\section{Introduction}

In recent years, multilevel power electronic inverters are finding increased attention in the design of large industrial electric drives in order to meet the high power demands required by them. The major advantages of multilevel inverters are the reduction in harmonic distortion of output voltage waveform with increase in number of levels and flexibility to use a set of batteries or fuel cells in any intermediate stages [1-3]. Even though multilevel inverters have made their way successfully to the industrial applications with a proven technology, failure of power electronic switches and its fault diagnosis is still a hot research topic of many researchers. In industrial applications, where safe and reliable operation is always expected, it is important to monitor the condition of power electronic switches in inverters. As the number of level increases, number of power electronic switches also increases which leads to increase in probability of failure of any switch and hence any such fault should be detected at the earliest in order to avoid the operation of drive and motor under abnormal conditions [4-14].

Among the various modes of failures of power electronic switches, open-switch and short-switch faults are most common and leads to current distortion and creates problems in gate drivers and hence reduces the system performance.

$\dagger$ Corresponding Author: Faculty. of Electrical Engineering, Anna University, Chennai, Tamilnadu, India. (parimalasundarpsg@gmail.com)

* Dept. of Electrical and Electronics Engg., Knowledge Institute of Technology, Salem, Tamilnadu, India (varmans03@gmail.com)

Received: October 14, 2014; Accepted: June 23, 2015
Some researchers used the inverter current [8-9] and inverter output voltage [11-15] to develop the fault diagnostic system. Surin Khomfoi et al. [13], developed an open-switch fault diagnostic system of a multilevel inverter using the output voltage FFT pattern and five parallel neural networks with 40 input neurons per network. Since the size of the neural network is high, in another paper, Surin Khomfoi et al., proposed an alternative methodology in which combination of FFT, principal component analysis, genetic algorithm and neural network approach was used to detect the fault type and fault location [14]. Identification of switch fault of multilevel inverters is still a hot research topic and many researchers are working hard to identify the fault accurately. However, reports on real time implementation of high performance fault diagnostic system for cascaded H-bridge multilevel inverter are scanty.

Considering the above facts, in this work, inverter output voltage is considered as an important parameter for faulty switch identification of multilevel inverter. Discrete Wavelet Transform (DWT) approach is used to extract important features such as energy content of output voltage waveform at different frequency bands [16-18]. Real time application of the proposed fault diagnostic system is implemented through LabVIEW software, which is a sophisticated tool for developing and running real time applications. LabVIEW utilizes graphical programming language developed by National Instruments and it has been successfully applied for data acquisition, instrument control and industrial automation [19-21]. Artificial Neural Network (ANN) is a powerful tool in the classification of patterns through learning and nonlinear mapping [22-23]. Deploying both 
LabVIEW and ANN is considered to be necessary as a further toolset for researchers. Hence, in this work, real time fault identification process is automated through LabVIEW based ANN approach.

\section{Architecture of H-Bridge Multilevel Inverter}

Cascaded multilevel inverter is composed of a number of $\mathrm{H}$-Bridge inverter cells connected in series in each phase with a separate DC source for each cell. Fig. 1 shows a typical $3 \phi$ cascaded multilevel inverter with three $\mathrm{H}$ bridge cells in each phase connected to a $3 \phi$ induction motor load. The number of levels in the output voltage waveform can be evaluated by $2 S+1$, where ' $S$ ' is the number of H-Bridge cells used in the system. Even though $3 \phi$ multilevel inverter system is commonly used in industrial drives, in the present work, $1 \phi$ multilevel inverter is only considered since the proposed diagnostic system can be extended for $3 \phi$ applications.

Fig. 2 shows the schematic of $1 \phi$ five level cascaded $\mathrm{H}-$ bridge inverter used in the present work consisting of two H-Bridge cells and eight IGBT switches. Each IGBT

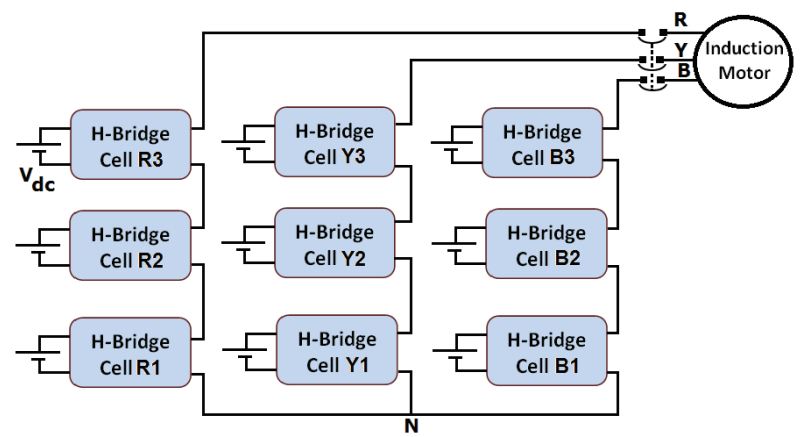

Fig. 1. Schematic of a typical $3 \phi$ cascaded H-Bridge multilevel inverter with induction motor load.

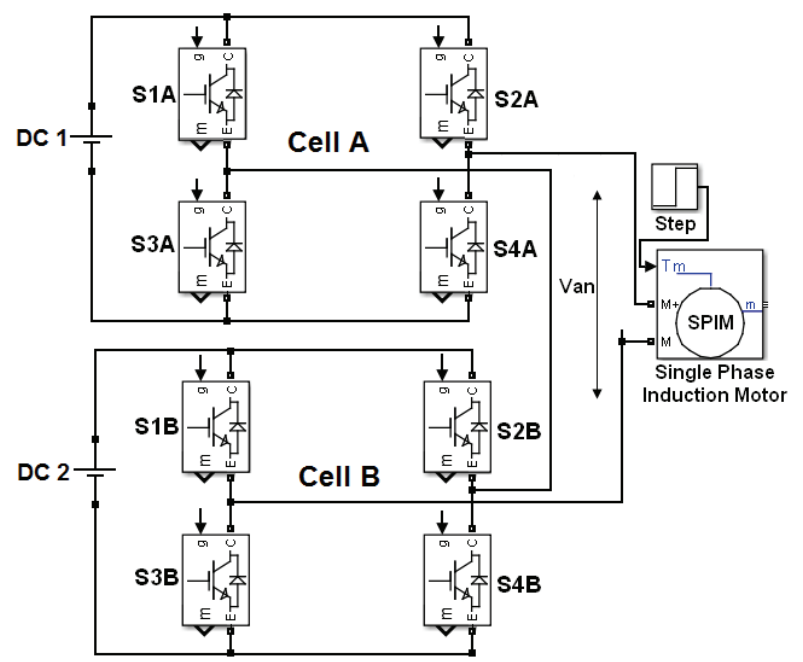

Fig. 2. Schematic diagram of $1 \phi$ cascaded H-Bridge five level inverter connected with induction motor load.

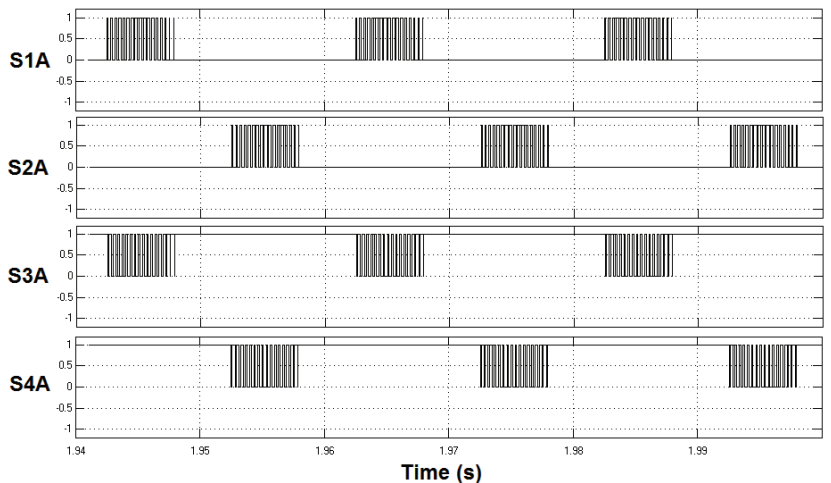

Fig. 3. Sinusoidal pulse width modulated switching pulses of cell A generated with carrier frequency $3 \mathrm{kHz}$ and modulation index 0.85 .

switch is labeled according to their cell position as S1A, $\mathrm{S} 1 \mathrm{~B}$, etc. Inverter is connected with a dynamic load of $1 \phi$, $0.5 \mathrm{HP}, 50 \mathrm{~Hz}$ induction motor.

Sinusoidal pulse width modulation (SPWM) technique is used to produce the required switching pulses of IGBT switches. In the case of SPWM, high frequency triangular carrier signal is compared with a reference sinusoidal signal. Fig. 3 shows the generation of trains of switching pulses of cell A at a carrier frequency $(f c)$ of $3 \mathrm{kHz}$ and modulation index $(m)$ of sinusoidal signal 0.85 . In the present work, $m$ is varied in the range of 0.8 to 0.95 .

\section{Analysis of Output Voltage and Current Pattern}

Simulation studies were carried out using Matlab Simulink software. In order to understand the output voltage and current pattern before and after the initiation of fault of multilevel inverter, initially open circuit fault is initiated at $1 \mathrm{sec}$ in the S1A switch of cell A. Fig. 4 shows the typical output voltage, its magnified view and current waveform before and after the fault of single phase cascaded H-Bridge multilevel inverter connected with induction motor load. Voltage and current signals are sampled at $20 \mathrm{kHz}$. Similarly, Fig. 5 shows the output voltage and current waveforms after the initiation of open circuit fault in S2A switch of cell A.

From the visual inspection of voltage and current waveforms, it is noticed that output voltage waveform pattern shows considerable variations for each fault, whereas it is difficult to classify the nature of fault based on the load current waveform. For example, after the initiation of open circuit fault in switch S1A and S2A, corresponding load current waveform seems to follow similar pattern and hence it is difficult to distinguish the faulty switch. In addition, load current is dependent on nature of load variations and hence it may lead to false diagnosis of nature of fault. Since the output voltage is independent of load and also shows distinguishable patterns for each switch fault, in the present work, output 

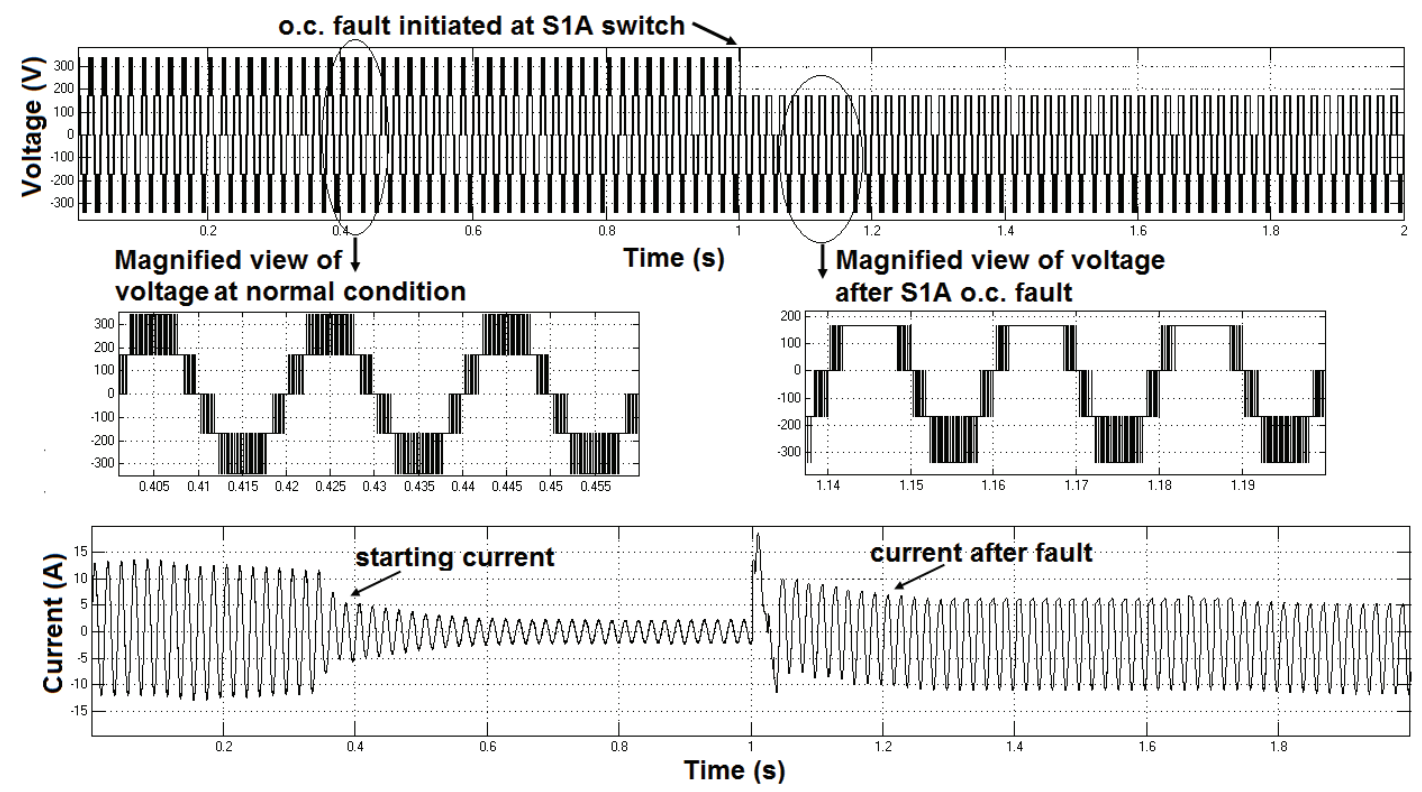

Fig.4. Output voltage and load current waveform before and after S1A open circuit fault.
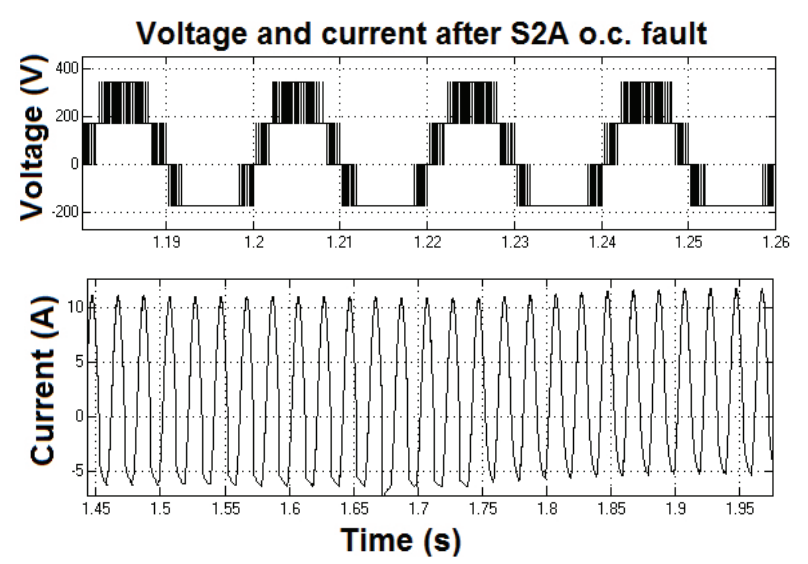

Fig.5. Output voltage and load current waveform after S2A open circuit fault.

voltage waveform is considered as an important parameter to develop the fault identification system.

\section{Concept of Discrete Wavelet Transform and Feature Extraction from Output Voltage Signal}

Extraction of salient features of the output voltage data, which in turn actively drives diagnostic knowledge out of the raw data, plays a major role in the novel fault diagnostic technologies. In order to develop an efficient fault diagnostic system, it is necessary to perform both time and frequency domain analysis of output voltage signals. DWT technique has been found to be efficient to extract features from the output voltage data. Multi resolution signal decomposition analysis (MRA) of DWT aims at ultimately producing a time-scale representation of the given discretized signal $\mathrm{x}(\mathrm{n})$ at various decomposition levels [15-18]. Let $\mathrm{c}_{0}[\mathrm{n}]$ be the original signal sequence. After convolution with $\mathrm{h}$ and $\mathrm{g}$ quadrature mirror filters, it is decomposed into an approximation component $\mathrm{c}_{1}[\mathrm{n}]$ and a detail component $d_{1}[n]$ at scale 1 . Then approximation component $c_{1}[n]$ is further decomposed into $c_{2}[n]$ and $d_{2}[n]$ at the next scale and so on. This type of hierarchical decomposition can be mathematically represented as,

$$
\begin{aligned}
& c_{m}[n]=\sum_{k} h[k-2 n] c_{m-1}[k] \\
& d_{m}[n]=\sum_{k} g[k-2 n] c_{m-1}[k]
\end{aligned}
$$

where $\mathrm{m}$ represents the scale of decomposition, $\mathrm{n}$ represents the sampling points and $\mathrm{k}$ represents translation coefficient. MRA is mainly used to achieve two important properties. The first is the localization property in time to identify any characteristic change. This will appear as large coefficients at the time of disturbance. The second is the partitioning of the signal energy into different frequency bands. This gives an idea of the frequency content in the distorted signal. It is well known that Daubechies 4 wavelet is very much useful in identifying any transition in the signal due to high frequencies and it is successfully applied as a mother wavelet in earlier papers [15-18]. Therefore Daubechies 4 wavelet has been chosen in the present analysis. Output voltage signals were decomposed up to 9 levels and the corresponding frequency band of detailed components is shown in Table 1. Standard deviation can be considered as a measure of the energy present in the signal with zero mean. Therefore, standard deviation values are calculated for detailed components to identify the transient energy present in the signal at 
Table 1. Frequency band of detailed components

\begin{tabular}{c|c}
\hline Detailed components of DWT & Frequency band $(\mathrm{Hz})$ \\
\hline D1 & $5000-10000$ \\
\hline D2 & $2500-5000$ \\
\hline D3 & $1250-2500$ \\
\hline D4 & $625-1250$ \\
\hline D5 & $312.5-625$ \\
\hline D6 & $156.25-312.5$ \\
\hline D7 & $78.125-156.25$ \\
\hline D8 & $39.0625-78.125$ \\
\hline D9 & $19.53125-39.0625$ \\
\hline
\end{tabular}

different level of decomposition (D1 to D9) [15-18]. Standard deviation of the $\mathrm{n}^{\text {th }}$ level of detailed signal is calculated using the formula,

$$
s t d=\sqrt{\frac{1}{N_{n}-1} \sum_{j=1}^{N_{n}}\left[d_{n}(j)-\mu_{n}\right]^{2}}
$$

where $\mu_{n}$ is the mean of the vector $d_{n}$ and $N_{n}$ is the length of the vector $d_{n}$. Energy content of the signal obtained from DWT MRA technique is considered as an important feature for the fault diagnosis of multilevel inverter system.

\subsection{Feature extraction from output voltage}

Fig. 6 shows DWT MRA detailed components of the output voltage signal at normal condition. The horizontal axis represents time (in samples) and the vertical axis represents magnitude of the DWT MRA detailed signals. Fig. 6 (D1-D9) shows the detailed component of DWT up to nine level of decomposition. Standard deviation of D1D9 detailed components are evaluated to understand the

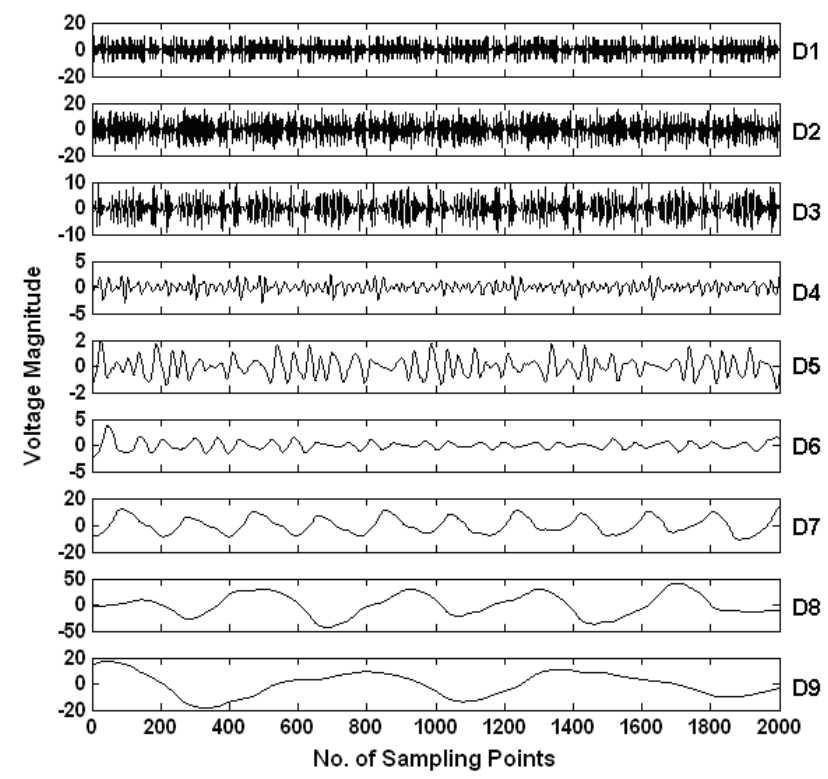

Fig.6. DWT MRA detailed components of output voltage waveform under normal condition obtained upto nine level of decomposition. ( $m=0.85 . f c=3 \mathrm{kHz})$.

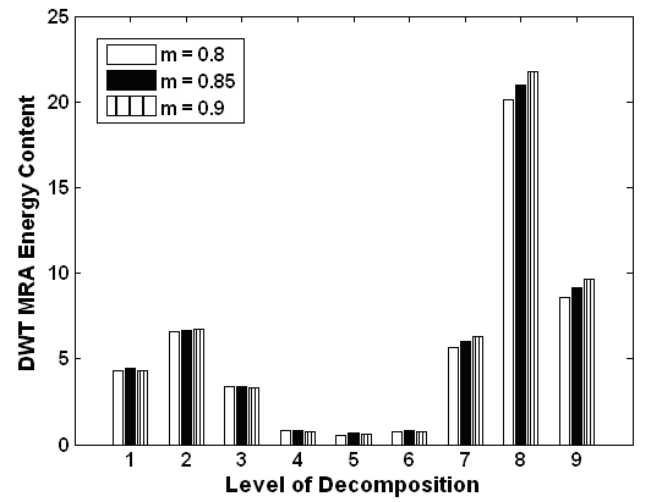

Fig. 7. DWT MRA energy content of output voltage waveform under normal condition obtained upto nine level of decomposition at different modulation index.

energy content of the signal at each level of decomposition [15-18].

The DWT MRA energy content of the voltage signal at different modulation index is shown in Fig. 7. Peak observed at $8^{\text {th }}$ level of decomposition corresponds to the $50 \mathrm{~Hz}$ fundamental component (Refer Table 1). Considerable increase in $7^{\text {th }}, 8^{\text {th }}$ and $9^{\text {th }}$ level of DWT MRA energy content of the signal with respect to increase in modulation index value is noticed. Similarly variations in the energy content of other frequency bands could be easily observed from Fig. 7.

\section{Structure of Fault Diagnostic System}

Fig. 8 shows the schematic of the overall fault diagnostic system developed for the identification of failure of power electronic switches in the multilevel inverter. It shows both hardware system and software system. Hardware system consists of the DC power supply, multilevel inverter, induction motor (IM) and data acquisition systems. Initially, both open and short switch faults are created by both simulation and experimental studies at different modulation index values and features are extracted from the voltage signal using DWT MRA analysis in Matlab. These features

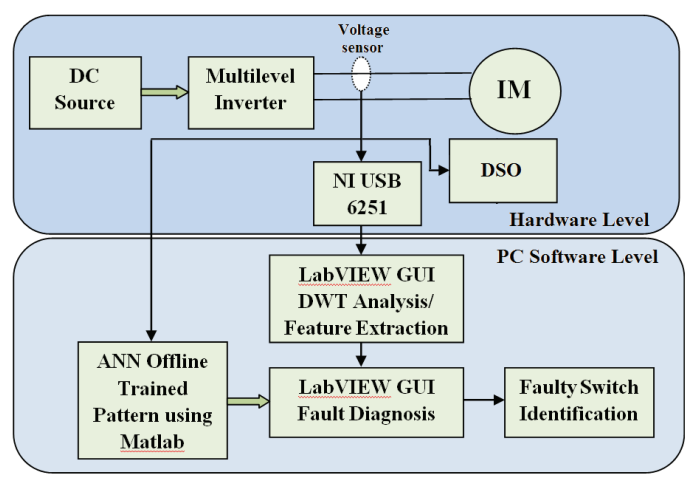

Fig. 8. Schematic of the overall fault diagnostic system. 
are given as an input to the ANN for offline training using Matlab. Trained pattern consisting of weight and bias values of the ANN is fed to the graphical programming language LabVIEW for fault diagnosis. In the case of real time application, voltage sensor output is given to NI USB data acquisition system which is connected to PC. Voltage data is processed in the LabVIEW DWT feature extraction analysis and compared with the offline trained pattern [21]. Then LabVIEW GUI indicates the faulty switch of the multilevel inverter, which is very useful for maintaining the reliability of the system.

Fig. 9 shows the photograph of laboratory experimental setup used to collect the output voltage signals of multilevel inverter at different switch fault conditions. Two H-bridge single phase PWM inverter modules are cascaded to get the five level output voltage waveform. IGBTs of rating $600 \mathrm{~V}, 25 \mathrm{~A}$ are used as a switching devices. PWM control module consisting of reference wave and carrier wave selection, modulation index and switching frequency adjustment is used to get the required gate pulses for the IGBT switches.

Fig. 10 shows the photograph of the data acquisition system interfaced with LabVIEW software in PC for real time applications. National Instruments (NI) USB-6251 (1.25 MSa/sec) is used as a data acquisition system and it is connected with PC for storing and further processing of signals. This system is capable of measuring 16 analog input signals, 16 bits. A digital storage oscilloscope, Agilent make $(1 \mathrm{GSa} / \mathrm{sec})$, is also used to visualize the output

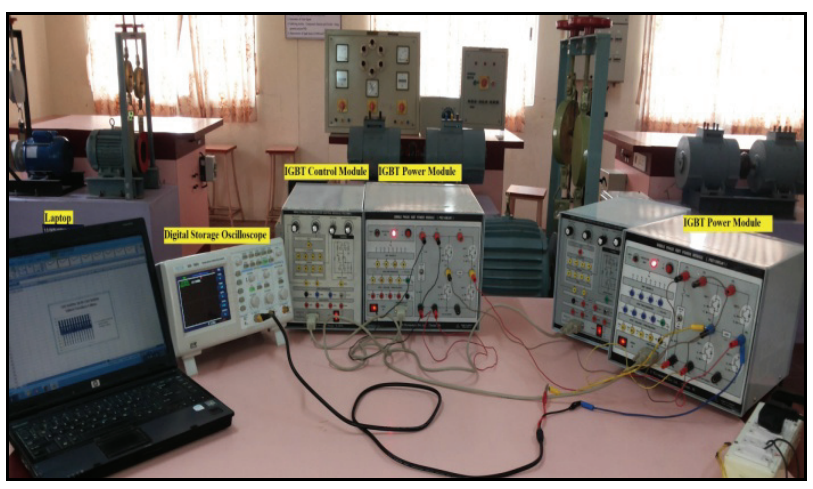

Fig.9. Laboratory experimental setup

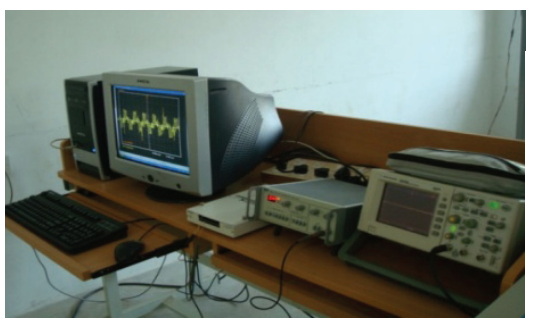

(a)

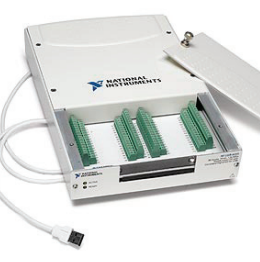

(b)
Fig. 10. (a) Data acquisition system interfaced with LabVIEW software in PC (b) NI USB-6251 hardware voltage waveforms. Open circuit and short circuit faults are created on each switch and corresponding output voltage waveform is recorded. Voltage sensor is used to collect the output voltage signals and it is connected with NI USB6251 . Output voltage signal is measured in the inverter at different modulation index values and DWT multi resolution signal decomposition is carried out. Important feature of the voltage signal i.e. energy content of the signal at different level of decomposition is extracted.

\subsection{Simulation results at open circuit fault}

Initially, open circuit fault is created on S1A switch and corresponding output voltage waveform is stored. Similarly, open circuit fault is created on all switches of both $\mathrm{H}$ Bridges $\mathrm{A} \& \mathrm{~B}$ and the corresponding voltage waveforms are stored for further feature extraction process. Fig. 11(i) shows the typical output voltage waveforms obtained at open circuit switch fault conditions of H-Bridge A. For comparison purpose, the voltage waveform obtained at normal (no fault) condition is also shown in the same figure. From the visual inspection of the output voltage waveforms, it is clear that it is possible to easily identify

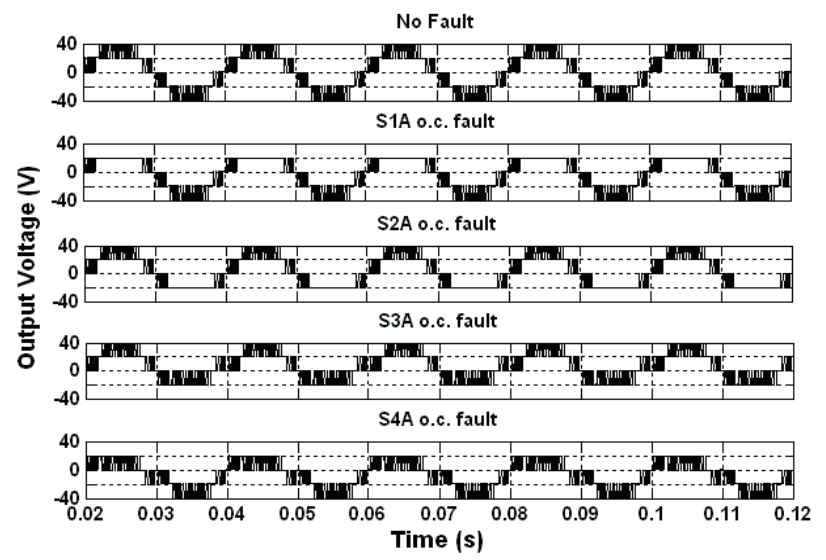

(i)

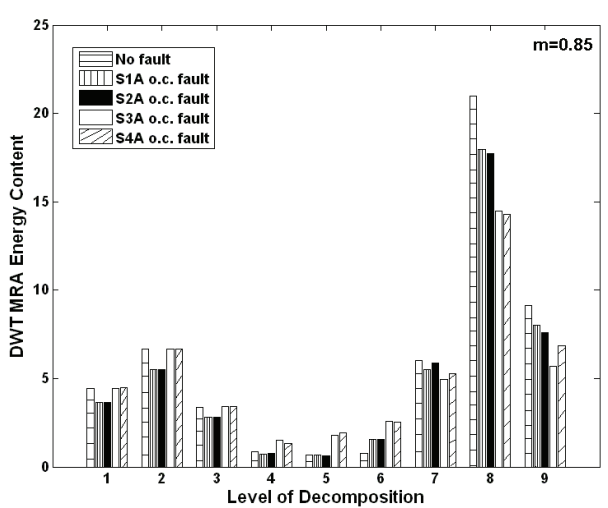

(ii)

Fig. 11. (i) Output voltage waveform obtained at different open circuit switch fault conditions of H-Bridge A (ii) corresponding DWT MRA energy content of output voltage waveforms. $(m=0.85 . f c=3 \mathrm{kHz})$. 
the variations under fault conditions with respect to normal condition.

However, in order to automate the process of fault diagnosis, it is important to extract distinct features from the output voltage waveform. Hence DWT MRA technique is applied to all the output voltage waveforms under open circuit fault conditions and the corresponding DWT MRA energy content is evaluated. Fig. 11(ii) shows the typical DWT MRA energy content of the voltage waveforms under open circuit switch fault conditions. It is observed that there is a slight variations in the energy content of the signal at open circuit fault conditions of different switches when compared with no fault condition. As expected, the energy content of the normal condition is higher when compared with open circuit fault conditions. Similarly, DWT MRA energy content is extracted at different modulation index values ranging from 0.8 to 0.95 and there exists considerable difference between different fault cases.

\subsection{Simulation results at short circuit fault}

In this case, short circuit fault is created on all switches of both H-Bridges A \& B one by one and corresponding output voltage waveform is stored for further feature extraction process. Fig. 12(i) shows the typical output

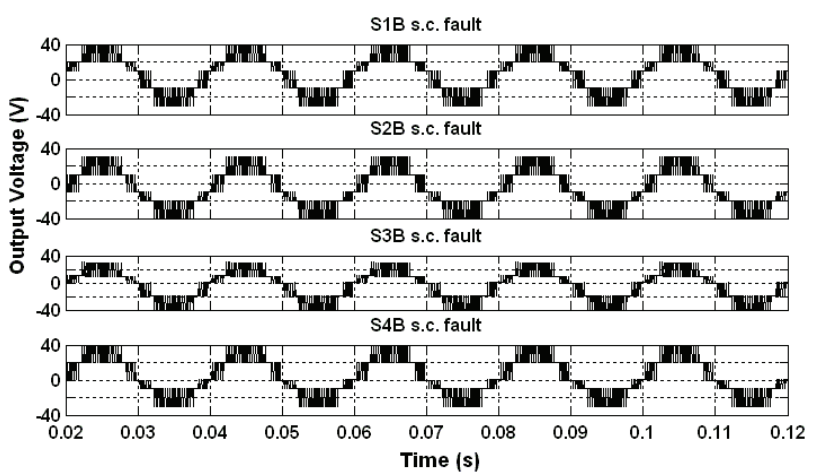

(i)

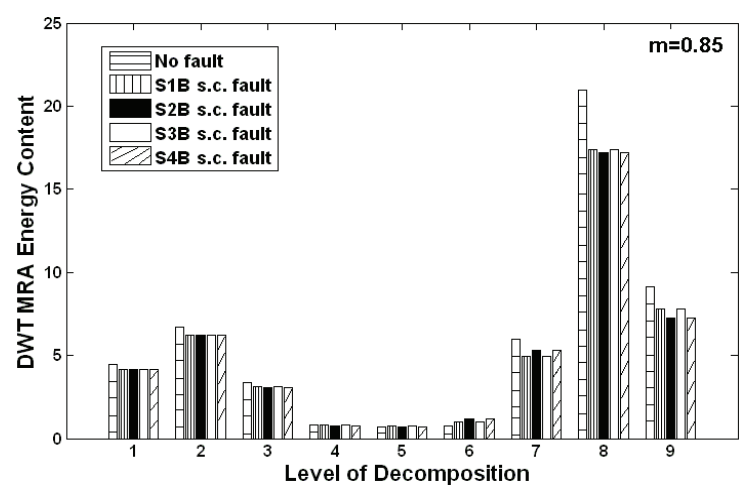

(ii)

Fig. 12. (i) Output voltage waveform obtained at different short circuit switch fault conditions of H-Bridge B (ii) corresponding DWT MRA energy content of output voltage waveforms. $\left(m=0.85 . f_{c}=3 \mathrm{kHz}\right)$. voltage waveforms obtained at normal (no fault) and shortswitch fault conditions of H-Bridges B. It is noticed that there is a considerable difference in all output voltage waveforms under short-switch fault conditions when compared with no fault condition.

DWT MRA technique is applied to all the output voltage waveforms under short circuit fault conditions and the corresponding DWT MRA energy content is evaluated. Fig. 12(ii) shows the typical DWT MRA energy content of the voltage waveforms under short circuit fault conditions. It is observed that there is a considerable variations in the energy content of the signal at levels $6,7,8$ and 9 when compared with other fault cases [15]. Similar to open circuit fault case, the energy content of the no fault condition is higher when compared with short circuit fault conditions.

\section{Experimental Validation}

For practical real time applications, it is essential to validate the features extracted from the simulation studies through laboratory measurements.

\subsection{Feature Extraction Analysis using LabVIEW}

The complete fault diagnostic system has been developed using LabVIEW 8.5 software. Fig. 13 shows the developed LabVIEW front panel consisting of output voltage capture and analysis module. The graphical user interface developed in LabVIEW displays the acquired output voltage waveforms in the front panel of the program. The front panel acts like a user interface where the user can input and extract data. Once the NI USB device is properly interfaced with this LabVIEW front panel, device communication indicator will blink in green. Front panel has control over

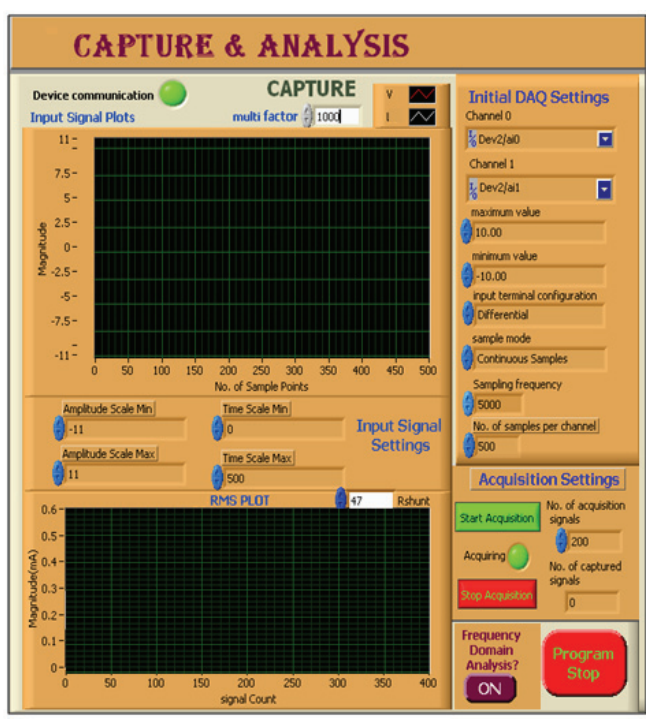

Fig. 13. LabVIEW front panel of the output voltage capture and analysis of multilevel inverter 


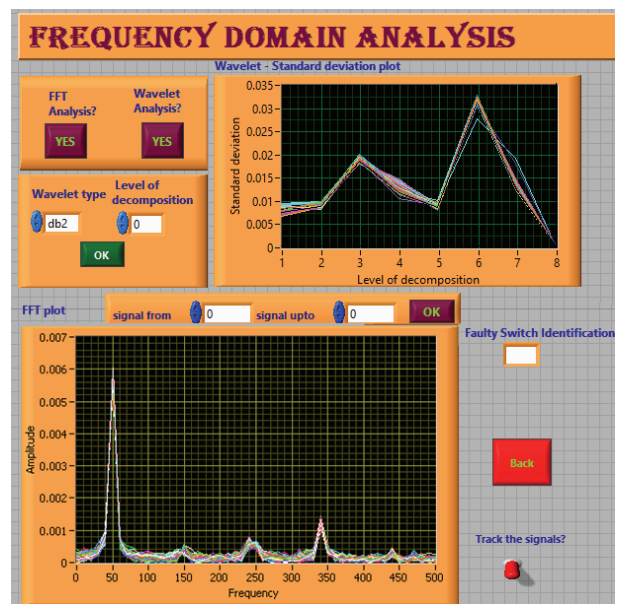

Fig. 14. LabVIEW front panel of frequency domain analysis and faulty switch identification
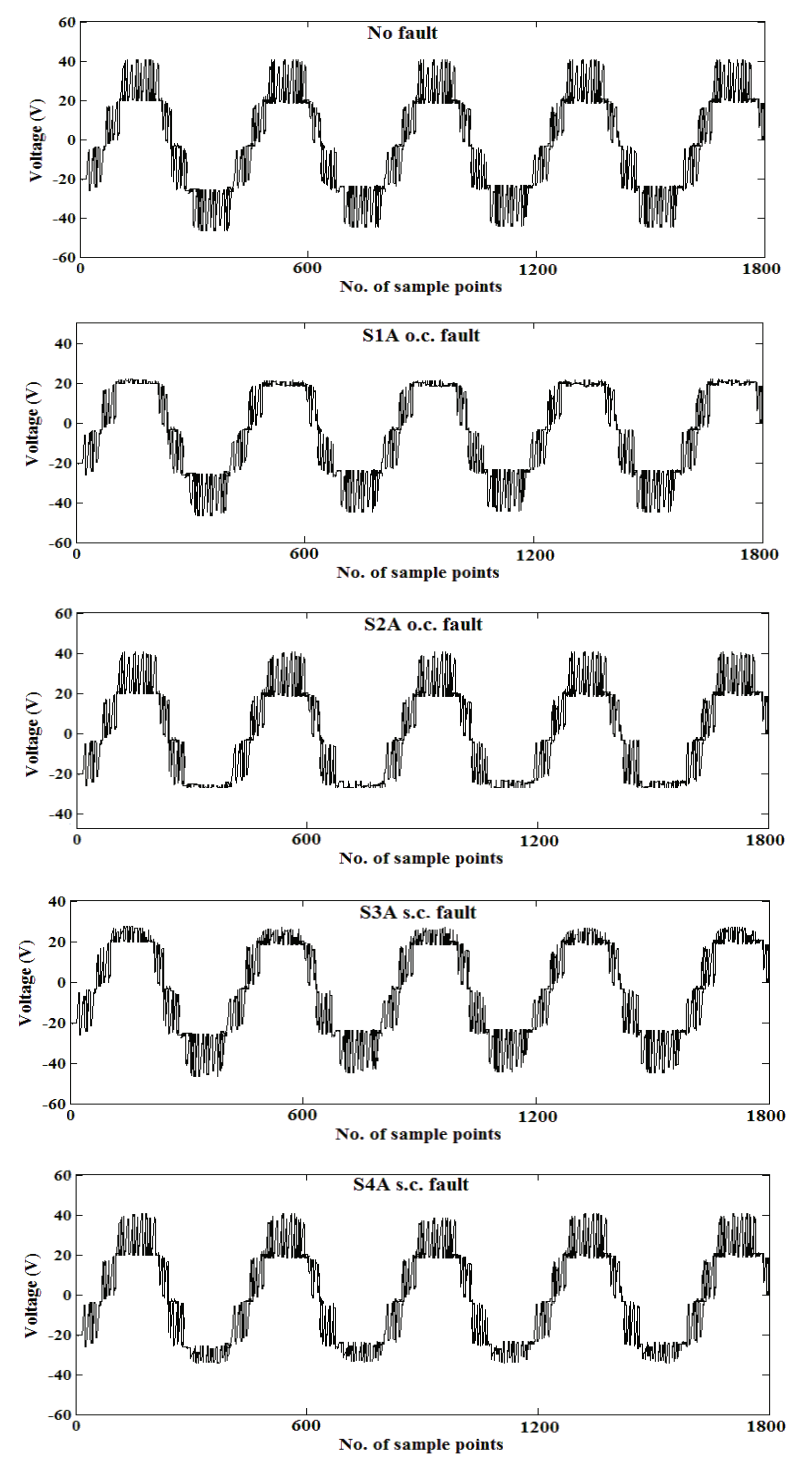

Fig. 15. Typical output voltage waveform obtained at different o.c and s.c switch fault conditions of $\mathrm{H}$ Bridge A during the experimental studies $(m=0.85)$

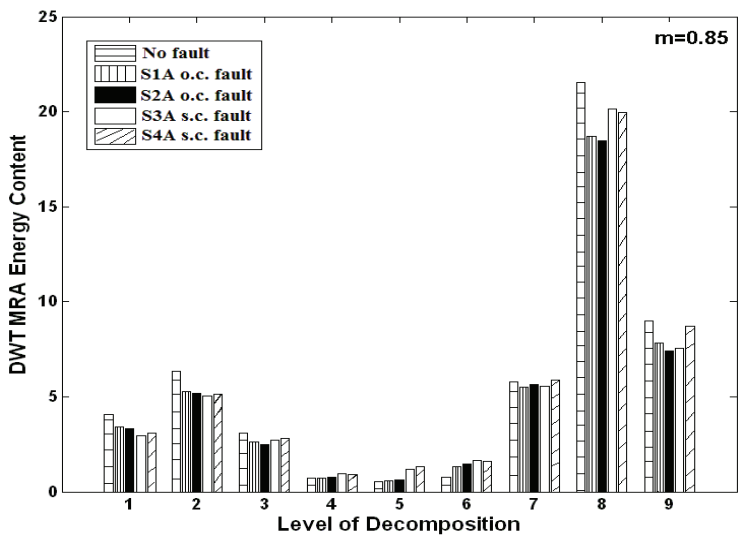

Fig. 16. Typical DWT MRA energy content of output voltage waveform at different fault conditions obtained during experimental studies. $(m=0.85)$.

output voltage signal parameters such as time scale, magnitude scale, sampling frequency and number of samples of each signal. Also, it is possible to set the number of signals to be captured at a particular time using the acquisition settings. Separate control is given for frequency domain analysis of voltage signals. Fig. 14 shows the front panel of LabVIEW developed for frequency domain analysis and faulty switch identification. This module consists of control over the selection of type of wavelet and number of level of decomposition. In this module, control is also given to track the DWT STD-MRA energy content variations of individual voltage signal. For comparison purpose, this module is also developed with FFT analysis. This module compares the real time voltage signal features with offline trained pattern and identifies the faulty switch.

Fig. 15 shows the output voltage waveforms measured during the experimental studies at different fault conditions and corresponding DWT MRA energy content features are shown in Fig. 16. It is noticed that there exists a close relationship between the output voltage waveform pattern obtained with experimental studies and simulation. DWT MRA energy content of the output voltage waveforms obtained during experimental studies looks similar to simulation studies and the difference in the DWT MRA energy content at each level of decomposition is also minimum.

\subsection{Real time fault diagnosis results from LabVIEW- ANN approach}

In order to automate the process of fault diagnosis of multilevel inverters, multilayer feed forward network (as shown in Fig. 17) with back propagation learning algorithm has been used [15, 22-23].

It consists of an input layer, one hidden layer and an output layer. The input and target vectors should be fed initially for training the network pattern. Training the network is done by changing the weights and biases of 


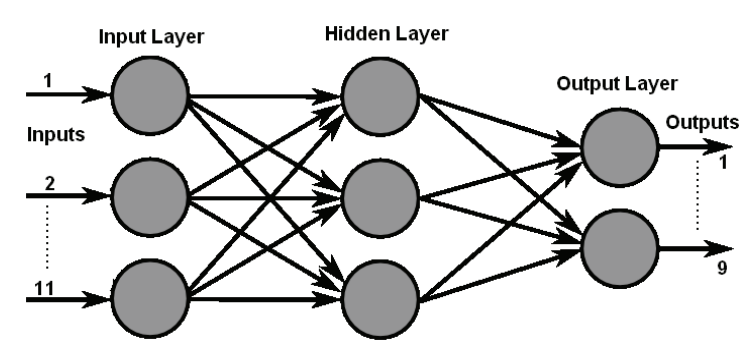

Fig.17. Schematic of artificial neural network.

the units depending on the resultant error. In the back propagation training algorithm, for each data set, forward pass and backward pass is carried out until the mean square error (MSE) is too low. The convergence is reached when the error between the measured and the desired output is less than fixed value (convergence criteria). The MSE, which is the average of sum of the errors for all set of inputs and corresponding outputs, is calculated as follows,

$$
M S E=\frac{1}{m} \sum_{k}^{m}\left(S_{k}-Y_{k}\right)^{2}
$$

where $S_{k}$ and $Y_{k}$ are respectively the desired and measured output for the $\mathrm{k}^{\text {th }}$ input set and $\mathrm{m}$ is the total number of input sets [15,22-23]. The details of the optimized neural network used in the present study are shown in Table 2.

Table 2. Specifications of ANN

\begin{tabular}{c|c}
\hline No. of inputs & 9 \\
\hline No. of neurons in hidden layer & 18 \\
\hline No. of neurons in output layer & 9 \\
\hline Learning rate $(\eta)$ & 0.1 \\
\hline No. of iterations & 2500 \\
\hline No. of training sets & 200 \\
\hline No. of test input sets & 150 \\
\hline Convergence criteria & 0.01 \\
\hline
\end{tabular}

In this work, the 9 values of energy content obtained from DWT MRA technique at different fault conditions are given as an input to the neural network. The 9 output neurons were used to classify the fault as no fault, S1A fault, S2A fault, S3A fault, S4A fault, S1B fault, S2B fault, S3B fault and S4B fault. Table 3 shows the ANN training pattern used for different switch faults of multilevel inverter.

Table 3. Training pattern of neural network

\begin{tabular}{|c|c|c|}
\hline Nature of Fault & Positon of Neuron & Output Pattern \\
\hline No fault & 1 & {$\left[\begin{array}{lllllllll}1 & 0 & 0 & 0 & 0 & 0 & 0 & 0 & 0\end{array}\right]$} \\
\hline S1A fault & 2 & {$\left[\begin{array}{lllllllll}0 & 1 & 0 & 0 & 0 & 0 & 0 & 0 & 0\end{array}\right]$} \\
\hline S1B fault & 3 & {$\left[\begin{array}{lllllllll}0 & 0 & 1 & 0 & 0 & 0 & 0 & 0 & 0\end{array}\right]$} \\
\hline S2A fault & 4 & {$\left[\begin{array}{llllllllll}0 & 0 & 0 & 1 & 0 & 0 & 0 & 0 & 0\end{array}\right]$} \\
\hline S2B fault & 5 & {$\left[\begin{array}{lllllllll}0 & 0 & 0 & 0 & 1 & 0 & 0 & 0 & 0\end{array}\right]$} \\
\hline S3A fault & 6 & {$\left[\begin{array}{lllllllll}0 & 0 & 0 & 0 & 0 & 1 & 0 & 0 & 0\end{array}\right]$} \\
\hline S3B fault & 7 & {$\left[\begin{array}{llllllllll}0 & 0 & 0 & 0 & 0 & 0 & 1 & 0 & 0\end{array}\right]$} \\
\hline S4A fault & 8 & {$\left[\begin{array}{llllllllll}0 & 0 & 0 & 0 & 0 & 0 & 0 & 1 & 0\end{array}\right]$} \\
\hline S4B fault & 9 & {$\left[\begin{array}{lllllllll}0 & 0 & 0 & 0 & 0 & 0 & 0 & 0 & 1\end{array}\right]$} \\
\hline
\end{tabular}

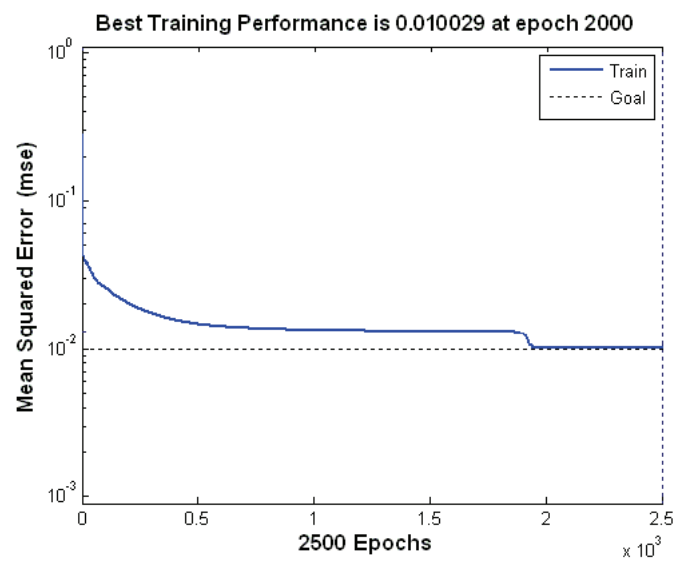

Fig. 18. Variations in the MSE of the ANN during training with respect to increase in no. of iterations.

The neural network offline training is performed with 200 training sets at different modulation index ranging from 0.8 to 0.95 . The convergence criteria is set as 0.01 . Fig. 18 shows the convergence of the mean square error near 2000 iterations. Thick line shows the variations of MSE during training and dotted line is the convergence criteria. It indicates that 2500 iterations are sufficient for the successful training of the optimized neural network. Then the trained network pattern consisting of weight and bias values of the ANN is transferred to LabVIEW graphical programming module for fault diagnosis.

For real time testing of the proposed system, 150 different fault cases at different modulation index ranging from 0.8 to 0.95 are created in the laboratory and the corresponding identification performance of the LabVIEW GUI is tested. Fig. 19 shows the identification rates of the proposed fault diagnostic system at different no. of hidden layer neurons. It is observed that the performance of the system is better for 18 hidden layer neurons when compared with other cases and the overall identification rate for all fault cases is $100 \%$ in this case and the system is able to identify the fault efficiently almost for all fault cases.

During training phase, each neuron is trained for a

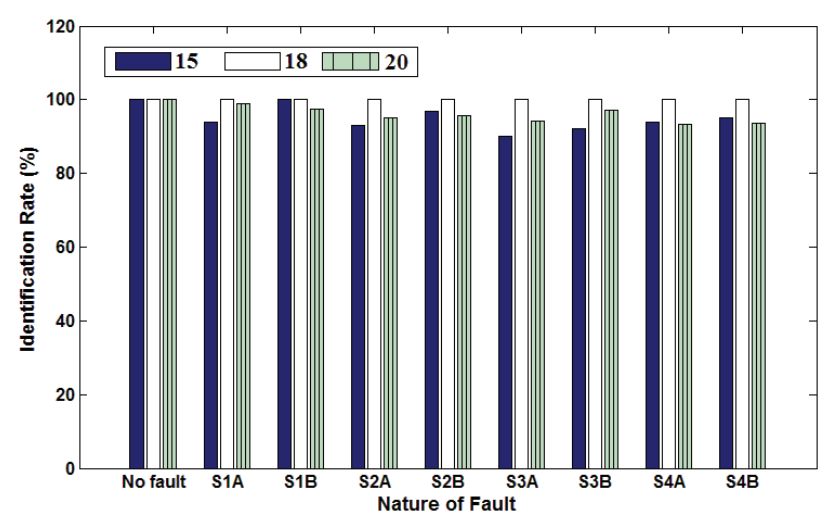

Fig.19. Identification rate of LabVIEW fault diagnostic system at different number of hidden layer neurons. 


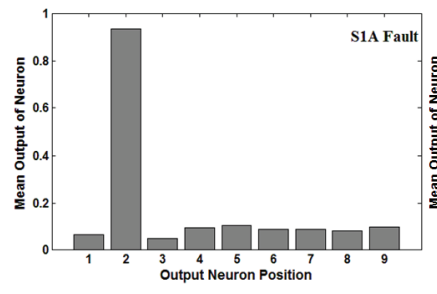

(a)

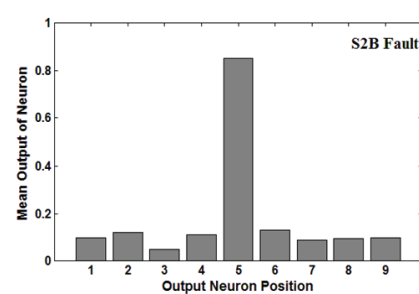

(c)

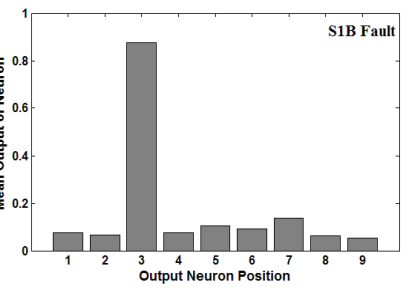

(b)

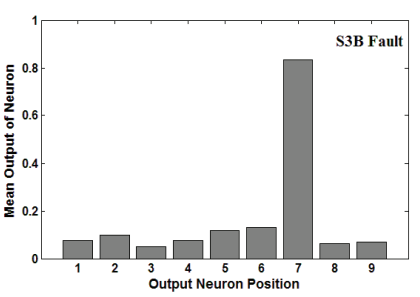

(d)
Fig. 20. Output of neurons in the LabVIEW fault diagnostic system: (a) S1A fault; (b) S1B fault; (c) S2B fault; (d) S3B fault.

binary number 0 or 1 as per the output pattern as shown in Table 3. For example, if the input training pattern corresponds to S1A fault, then the second neuron in the output layer is trained for 1 and all other neurons are trained for 0. During real time testing using LabVIEW fault diagnostic system, when an untrained input pattern corresponding to S1A fault is given as an input, then the output of second neuron should be maximum when compared with other neurons. Fig. 20 shows the output of the neurons in the LabVIEW fault diagnostic system for different fault cases. It is observed that the output of neuron corresponding to that fault position in most of the case is above 0.8 . It is easier to differentiate the output neuron corresponding to faulty switch from remaining output neurons. This indicates that the trained neural network can assess the fault condition of the multilevel inverter accurately. In the present work, the high speed NI USB 6251,16 bit, $1.25 \mathrm{MSa} / \mathrm{sec}$ data acquisition system is connected with a laptop which has intel core i5 processor with a clock speed of $2.7 \mathrm{GHz}$ and $4 \mathrm{~GB}$ RAM. In this approach, the LabVIEW GUI takes 10 milliseconds to diagnose the faulty switches.

The reported real time LabIEW based testing of fault diagnosis of multilevel inverter using output voltage characteristics such as DWT MRA energy content shows that it is possible to identify the failure of specific switch of multilevel inverter accurately. When compared with the techniques reported in earlier papers [13-14], proposed method drastically reduces the number of inputs to the ANN network and diagnoses the fault cases in 10 milliseconds. As the number of inputs to ANN reduces, it makes faster training process. Also proposed method determines the failure of specific switch (may be due to open-switch fault or short-switch fault) of multilevel inverter. In addition, proposed technique provides $100 \%$ identification rate between normal and faulty condition. Hence, once the faulty switch is diagnosed, it will be useful for the operator to carryout preventive maintenance work.

\section{Conclusion}

Faulty switch diagnosis of a cascaded H-bridge five level inverter connected with an induction motor load is studied in this work. Salient features of the output voltage waveform are investigated by both simulation and experimental studies at different open-switch and shortswitch fault cases. Important feature such as energy content of the signal obtained using DWT technique is given as an input to the back propagation trained neural network. Matlab software is used to perform offline training of neural network. Real time application of the proposed fault diagnostic system was implemented through the LabVIEW software. In the proposed fault diagnostic system, it is possible to exactly identify the individual faulty switch. This system is able to classify $100 \%$ accurately the normal and faulty conditions. Hence, once switch fault is identified, it will be useful for the operator to carryout preventive maintenance work.

\section{References}

[1] Zedong Zheng, Kui Wang, Lie Xu and Yongdong Li, "A hybrid cascaded multilevel converter for battery energy management applied in electric vehicles", IEEE Trans. Power Electron., vol. 29, no. 7, pp. 35373546, Jul. 2014.

[2] Javad Gholinezhad and Reza Noroozian, “Analysis of Cascaded H-Bridge Multilevel Inverter in DTCSVM Induction Motor Drive for FCEV", J Electr Eng Technol, vol. 8, no. 2, pp. 304-315, 2013.

[3] M. R. Banaei and E. Salary, "A New Family of Cascaded Transformer Six Switches Sub-Multilevel Inverter with Several Advantages", J Electr Eng Technol, vol. 8, no. 5, pp. 1078-1085, 2013.

[4] Ui-Min Choi, Kyo-Beum Lee and Frede Blaabjerg, "Diagnosis and tolerant strategy of an open-switch fault for T-type three-level inverter systems", IEEE Trans. Ind. Appl., vol. 50, no. 1, pp. 495-508, Jan./Feb. 2014.

[5] A. Chen, L. Hu, L. Chen, Y. Deng, and X. He, “A multilevel converter topology with fault-tolerant ability", IEEE Trans. Power Electron., vol. 20, no. 2, pp. 405-415, Mar. 2005.

[6] Pablo Lezana, Josep Pou, Thierry A. Meynard, Jose Rodriguez, Salvador Ceballos and Frédéric Richardeau, "Survey on fault operation on multilevel inverters", IEEE Trans. Ind. Electron., vol. 57, no. 7, pp. $2207-$ 2218, Jul. 2010.

[7] Mingyao Ma, Lei Hu, Alian Chen and Xiangning He, 
"Reconfiguration of carrier-based modulation strategy for fault tolerant multilevel inverters", IEEE Trans. Power Electron, vol. 22, no. 5, pp. 2050-2060, Sep. 2007

[8] D. Diallo, M. H. Benbouzid, D. Hamad and X. Pierre, "Fault detection and diagnosis in an induction machine drive: A pattern recognition approach based on concordia stator mean current vector," IEEE Trans. Energy Conv., vol. 20, no. 3, pp. 512-519, Sep. 2005.

[9] J. Estima and A. Marques Cardoso, "A new algorithm for real-time multiple open-circuit fault diagnosis in voltage-fed PWM motor drives by the reference current errors", IEEE Trans. Ind. Electron., vol. 60, no. 8, pp. 3496-3505, Aug. 2013.

[10] M. A. S. K. Khan and M. A. Rahman, "Development and implementation of a novel fault diagnostic and protection technique for IPM motor drives", IEEE Trans. Ind. Electron., vol. 56, no. 1, pp. 85-92, Jan. 2009.

[11] P. Lezana, R. Aguilera and J. Rodriguez, "Fault detection on multicell converter based on output voltage frequency analysis," IEEE Trans. Ind. Electron., vol. 56, no. 6, pp. 2275-2283, Jun. 2009.

[12] M. Abul Masrur, Z. Chen, and Y. Murphey, "Intelligent diagnosis of open and short circuit faults in electric drive inverters dfor real-time applications", IET Power Electron., vol. 3, no. 2, pp. 279-291, Mar. 2010.

[13] Surin Khomfoi and L. M. Tolbert, "Fault diagnostic system for a multilevel inverter using a neural network", IEEE Trans. Power Electron., vol. 22, no. 3, pp. 1062-1069, May 2007.

[14] Surin Khomfoi and L. M. Tolbert, "Fault diagnosis and reconfiguration for multilevel inverter drive using AI-based techniques", IEEE Trans. Ind. Electron., vol. 54, no. 6, pp. 2954-2968, Dec. 2007.

[15] M. Sivakumar, RMS. Parvathi, S. Chandrasekar, "Diagnostic Study of Short-Switch Fault of Cascaded H-Bridge Multilevel Inverter using Discrete Wavelet Transform and Neural Networks", International Journal of Applied Engineering Research, vol. 9, no. 21, pp. 10087-10106, 2014.

[16] R Sarathi, S Chandrasekar, C Venkataseshaiah, N Yoshimura, "Condition monitoring of outdoor polymeric insulation structures using wavelets and neural networks", IEEE Conference on Electrical Insulation and Dielectric Phenomena, pp.398-401, 2003.

[17] R. Sarathi, S. Chandrasekar and N. Yoshimura, "Investigations into the surface condition of the silicone rubber insulation material using multiresolution signal decomposition", IEEE Trans. Power Delivery, vol. 21, pp. 243-252, Jan. 2006.

[18] S.Chandrasekar, C.Kalaivanan, Andrea Cavallini and Gian Carlo Montanari, "Investigations on leakage current and phase angle characteristics of porcelain and polymeric insulator under contaminated condi- tions", IEEE Trans. Dielectr. Electr. Insul., vol. 16, no. 2, pp. 574-583, Apr. 2009.

[19] Sachin sharma and Gaurav kumar, "Object classification through perceptron model using LabVIEW", International Journal of Electronics and Communications Technology, vol. 2, no. 3, pp. 255-258, 2011.

[20] J. Jerome, Aravind, Arunkumar and Balasubramanian, "LabVIEW based Intelligent Controllers for Speed Regulation of Electric Motor", Proceedings of IEEE Instrumentation and Measurement Technology Conference, IMTC 2005. vol. 2, pp. 935-940, May 2005

[21] Nagarani, Nithyavathy and Parameshwaran, "Low cost Mobile Robot using Neural Networks in Obstacle Detection", International Journal of Scientific \& Engineering Research, vol.4, no.5, pp.26-29, May 2013.

[22] V. Jayaprakash Narayanan, B. Karthik and S. Chandrasekar, "Flashover Prediction of Polymeric Insulators Using PD Signal Time-Frequency Analysis and BPA Neural Network Technique", J Electr Eng Technol,vol. 9, no. 4, pp. 1375-1384, Feb 2014.

[23] S.Chandrasekar, "Wavelet Transform and Artificial Neural Network Approach to Infer the Surface Condition of Power Transmission Line Ceramic Insulators", Iranian Journal of Electrical And Computer Engineering, vol. 9, no. 1, pp. 59-66, 2010.

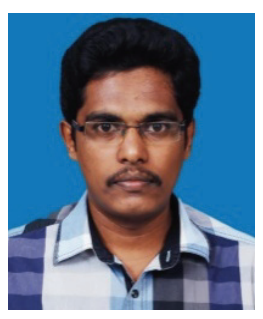

E. Parimalasundar obtained his B.E., Electrical and Electronics Engineering, Government College of Engineering, Bargur in 2007 from Anna University. He received his M.E., Power Electronics and Drives, PSG College of Technology, Coimbatore in 2009 from Anna University. He is presently pursuing toward his Ph.D., Electrical Engineering, Anna University, Chennai. He is also working as an Assistant Professor in the Department of Electrical and Electronics Engineering at Adhiyamaan College of Engineering, Hosur, Tamilnadu, India. His research interests include in the area of DC - DC Converters, AC - AC Converters, Multilevel Inverters, Soft Switching Techniques in Power Electronics and Drives.

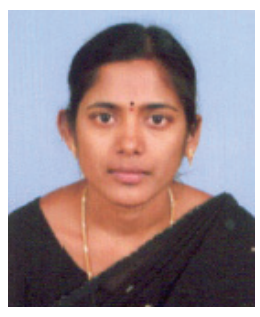

N. Suthanthira Vanitha obtained her B.E., Electrical and Electronics Engineering from Madras University. She received her M.E., Applied Electronics from Madurai Kamaraj University. She was awarded with Ph.D from Anna University, Chennai in the specializetion of Information and Communication 
Engineering. She is presently working as a Professor \& Head in the Department of Electrical and Electronics Engineering, Knowledge Institute of Technology, Salem, Tamilnadu, India. She also published about 40 journals at International level and 10 journals at National level. She had guided a student for getting internship from IIT Bombay in the idea of Medical shop explorer in e-yantra IDEAS competition. She is life member of ISTE \& CSI. Her research interests include in the area of Robotics, DSP, MEMS and Biomedical, Embedded Systems, Power Electronics and Renewable Energy System. 